\title{
Database Design for Aperture Array Hardware Management
}

\author{
D. Price and S. Schediwy
}

Department of Astrophysics, The University of Oxford, Keble Rd, Oxford, United Kingdom, OX1 3RH

\begin{abstract}
As the size and complexity of an aperture array grows, so too does the amount of hardware involved. As we approach SKA sized arrays of many thousands of elements, hardware management and characterisation will become an issue. As part of the SKADS programme, we have developed a simple hardware management database "pathfinder", which relates each hardware component to corresponding characterisation measurements and signal information. This database was used to store information about the analogue characterisation campaign conducted on the 2-PAD aperture array in the first half of 2009 (Price et al. 2009). This measurement database is available online at http://2-pad.physics.ox.ac.uk.
\end{abstract}

\section{Introduction}

2-PAD (the Dual Polarisation All-Digital aperture array), is a fully functional radio telescope demonstator, designed to operate within the $0.3 \mathrm{GHz}$ to $1.0 \mathrm{GHz}$ radio band (Harris et al., 2009). 2-PAD can be considered a technology pathfinder toward the Square Kilometre Array telescope (Taylor, 2007 and Greenwood, 2007). Due to the large number of components utilised in the 2-PAD analogue chain, there is need of a database to store measurement data. This database needs to be scalable; while we are currently working toward implementing a $4 \times 4 \times 2$ array, the long term goal is to implement the full $8 \times 8 \times 2$ system at Jodrell Bank. This upgrade would see the number of signal paths increase by a factor of 4 , from 32 to 128 . The number of analogue components will also grow by this factor, from $\sim 300$ for the $4 \times 4 \times 2$ array, to $\sim 1500$ for an $8 \times 8 \times 2$ array. To accommodate this expansion, we have created a relational database which links together serial numbers, signal IDs and measurements in a logical fashion. This measurement database is available online at http://2-pad.physics.ox.ac.uk.

If we were to extend the current 2-PAD design to the proposed size of a SKA substation (ie. up to $\sim 75000$ elements, see Faulkner, 2009), then the number of analogue components required could conceivably grow by over three orders of magnitude to over $1 \times 10^{6}$. While 2-PAD is of course not intended to be scaled by such a huge factor, this stills highlights the need to invest time into database design for SKA hardware management. The 2-PAD measurement database can be seen as a pathfinder toward future hardware management systems for the SKA.

The design of the 2-PAD database is discussed below in Section 2. Interface design is discussed in Section 3, and scalability toward SKA sized arrays is treated in Section 4.

\section{Database Design}

The 2-PAD measurement database is designed and built in MySQL 5, which is relational database software developed and supported by Sun Microsystems. MySQL is widely used in internet applications and is distributed as part of the open-source

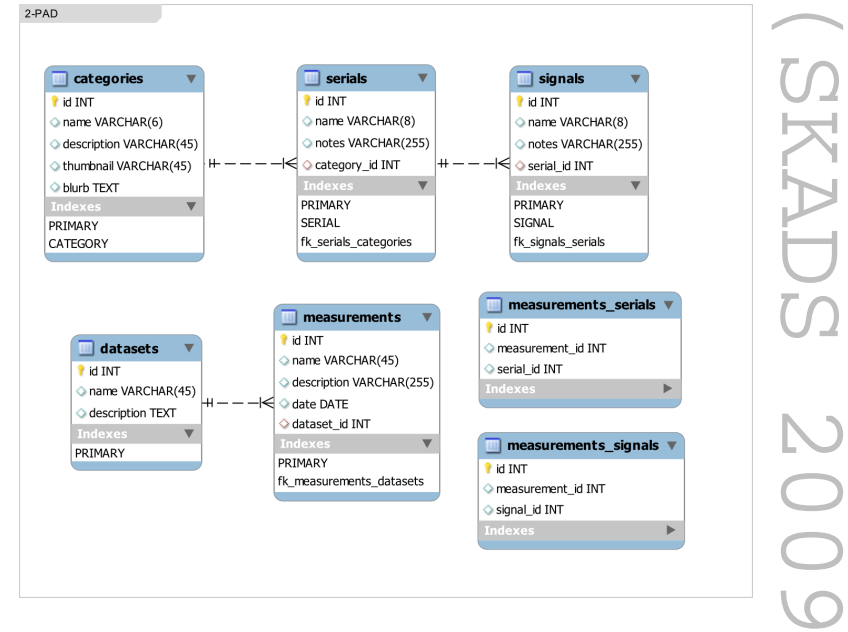

Fig. 1: The entity-relationship diagram for the 2-PAD database. The database design follows CakePHP nomenclature guidelines, which ensures CakePHP's automatic Object-Relational Mappings (ORM) function nominally.

server software stack, LAMP (Linux, Apache, MySQL, PHP / Perl / Python).

The 2-PAD MySQL database design is shown in the EntityRelationship (E-R) diagram of Figure 1. The arrows in the figure represent one-to-many relations between database tables. Data is retrieved from the database using Structured Query Language (SQL), which is generated automatically by the CakePHP frontend discussed in Section 3 below.

As shown in Figure 1, the categories and dataset tables can be considered the "superordinate" tables of the 2-PAD measurement database. The categories ${ }^{\text {a }}$ table stores general information about the different classes of analogue components (i.e. amplifiers, gain chain modules, coaxial cables, etc). The categories table has a one-to-many relation with the serials table, which stores information about each analogue component that has been assigned a serial number. This in turn has a one-to-

a While the term components may be more semantically accurate than categories, "components" is a reserved word in CakePHP so could not be used. 
many relationship with the signals table, which stores signal path information. This structure allows for a single component to have multiple related signal paths, as is the case with many of the components.

The other superordinate table, datasets, stores information about the measurement procedure, such as any laboratory working notes. The datasets table has a one-to-many relationship with the measurements table, which stores information about each measurement conducted. As the VNA outputs data as Comma Seperated Value (CSV) files, the measurements themselves are stored in a flat-file database. Each CSV file has a corresponding entry in the measurements table of the MySQL database, of which the name field is equivalent to the CSV filename; the naming scheme is discussed in detail in Price et al. (2009). The 2-PAD interface detailed below in Section 3 automatically retrieves and displays these $\mathrm{CSV}$ data.

\section{Interface Design}

We have designed an internet browser based user interface, to allow 2-PAD users to quickly access information and measurement data, as well as allowing technicians to document any changes to the system as they occur (such as upgrades to components, or replacing broken items). The interface is built in the CakePHP framework, which utilises MVC (Model-ViewController), architectural patterns, allowing for rapid development of internet applications. As the interface is browser based, any standards compliant internet browser can be used to access the database (such as Internet Explorer 7+, Mozilla Firefox $3+$, or Google Chrome 2+). As such, the database is accessible from virtually any computer, without the need for software installation.

To display the measurement data, the CSV files are read into an array by PHP scripts, then displayed graphically using the JpGraph library. Users also have the option of downloading these CSV files, if they wish to conduct further data analysis offline.

The 2-PAD measurement database also has a secure administration area, where users can add, edit and delete database entries. In addition to this, as the 2-PAD measurement database is an online application, we have integrated a website with general information about the 2-PAD project to further promote public awareness of the project. The 2-PAD website could easily be extended to provide community building resources, news feeds, forums and collaborative tools. The 2-PAD measurement database can be found online at http://2-pad.physics. ox.ac.uk.

\section{Scalability}

The 2-PAD measurement database has been designed to accommodate expansions and upgrades to the 2-PAD system. While 2-PAD aims to implement an $8 \times 8 \times 2$ array, the measurement database could easily accommodate for a $16 \times 16 \times 2$ array, or even $32 \times 32 \times 2$ array without approaching the table size limitations of mySQL.

The 2-PAD measurement database would not, however, scale to the size required to store the components of an SKA station, for which a more robust database solution would be advisable. It should be noted that an "SKA sized" database could potentially require a dedicated server and commercial database software, which would have both one-off costs in implementation, and ongoing costs in power, maintenance and administration. As such, we would suggest hardware management databases are considered in future SKA costing models and that further groundwork is done toward hardware management in aperture array systems as part of the AAVP.

Acknowledgements. We would like to thank Adam Baird, Tim Ikin and Richard Whitaker for their help and knowledge on the analogue components of 2-PAD. 2-PAD is a joint project of The University of Oxford, The University of Manchester and The University of Cambridge. 2-PAD has been developed under the Square Kilometre Array Design Studies project, which is funded by the European Commission Sixth Framework Programme and the United Kingdom Science and Technology Facilities Council.

\section{References}

CakePHP: the rapid development PHP framework. 2009

http: //www . cakephp.org

Faulkner A., 2009, "Aperture arrays in the SKA," In SKA Cape Town 2009.

Greenwood, C. "Status of Pathfinder Telescopes and Design 2 Studies," SKA Memos. 2007

Harris, G., et al., July 2009, "2-PAD: The New Arrival," SKADS IAU Newsletter. available at http://www. skads-eu.org/PDF/SKADSnewsletter05_IAU.pdf

MySQL :: About MySQL. 2009

http://www . mysql . com/about/

Price, D., Schediwy, S., Ikin, T., Jones, M., "The 2 PAD Analogue System: Characterisation, Commissioning, and Implications for Calibration and Beamforming,' SKA Memo \#122, 2010, available from http://www. skatelescope.org/pages/documents_3.htm

Taylor, A., 2007, The Square Kilometer Array. Proceedings $\square$ IAU Symposium No. 248. 